\title{
Netrin-1 Overexpression Protects Kidney from Ischemia Reperfusion Injury by Suppressing Apoptosis
}

\author{
Weiwei Wang, ${ }^{*}$ William Brian Reeves, ${ }^{*}$ \\ Laurent Pays, ${ }^{\dagger}$ Patrick Mehlen, ${ }^{\dagger}$ and \\ Ganesan Ramesh* \\ From the Division of Nephrology," Pennsylvania State University \\ College of Medicine, Hershey, Pennsylvania; and the Apoptosis, \\ Cancer, and Development Laboratory, ${ }^{\dagger}$ Equipe Labellisée la \\ Ligue, University of Lyon, France
}

Netrin-1, a diffusible laminin-related protein, is highly expressed in the kidney. However, the pathophysiological roles of netrin-1 in the kidney are unknown. To address this question directly, we used transgenic mice that overexpress chicken netrin- 1 in the kidney. Netrin-1 overexpression was confirmed by real-time RT-PCR and Western blot analysis. Eightweek-old wild-type and transgenic mice were subjected to 26 minutes of renal ischemia followed by reperfusion for 72 hours. Wild-type mice developed more severe renal dysfunction by 24 hours than netrin-1 transgenic mice. Functional improvement was associated with better preservation of morphology, reduced cytokine expression, and reduced oxidative stress in the kidney of transgenic mice as compared with wild-type mice. In addition, both basal and reperfusion-induced cell proliferation were dramatically increased in transgenic kidneys as determined by Ki-67 staining. Interestingly, ischemia reperfusion induced a large increase in apoptosis in wild-type mice but not in netrin-1 transgenic mice that was associated with reduced caspase- 3 activation in the transgenic kidney. These results suggest that netrin-1 protects renal tubular epithelial cells against ischemia reperfusion-induced injury by increasing proliferation and suppressing apoptosis. (Am J Pathol 2009, 175:1010-1018; DOI: 10.2353/ajpath.2009.090224)

Netrin-1, a diffusible laminin-related protein, is highly expressed outside the nervous system; its most abundant expression is found in the kidney. However, the function of netrin-1 in tissues outside the nervous system is not clear. In recent studies using both in vitro and in vivo systems, netrin-1 was shown to play a role in angiogenesis, ${ }^{1-3}$ cell migration, ${ }^{4}$ tissue morphogenesis,,${ }^{5,6}$ tumor progression and growth, ${ }^{7,8}$ and regulation of inflammation. ${ }^{9}$ Our most recent studies showed that administration of recombinant netrin-1 before renal ischemia reperfusion $(\mathrm{I} / \mathrm{R})$ injury prevented renal dysfunction and inflammation. ${ }^{10}$ Our studies also showed that netrin-1 protein was rapidly upregulated in renal tubular epithelial cells and could be detected in urine. However, the function of netrin-1 in tubular epithelial cells and how netrin-1 reduces renal dysfunction is not known.

Netrin-1 has a major role during the development of the nervous system by mediating chemo-attraction and chemo-repulsion. However, netrin-1 has also been described as a survival factor. Indeed, netrin-1 prevents cell death by acting as a ligand of the dependence receptors DCC and UNC5H. ${ }^{11-13}$ Netrin-1 binds to two families of receptors: DCC (DCC and neogenin) and $\mathrm{UNC5H}$ (UNC5A, UNC5B, UNC5C, and UNC5D). Netrin receptors are referred to as dependence receptors because these receptors have been shown to induce apoptosis in the absence of ligand (netrin). ${ }^{14}$ DCC, UNC5A, UNC5B, and UNC5C are all cleaved by caspase-3 in vitro, and mutation of the cleavage site strongly inhibits cell death in vitro and in vivo. ${ }^{12}$ However, the mechanism for DCC or UNC5-induced apoptotic signaling is still largely unknown. Recently, the administration of netrin-1 to mice before I/R of the kidney and the brain was shown to suppress tissue injury. ${ }^{10,15}$

To determine the role of netrin-1 in renal tubular epithelial cell function and the mechanism of netrin- 1 mediated protection against I/R injury of the kidney, we used mice that overexpress netrin-1 in the proximal tubular epithelial cells. Our results show that netrin- 1 transgenic

Supported by Beginning Grant-in-Aid from the American Heart Association and a Research Startup grant from the Department of Health of the Commonwealth of Pennsylvania to G.R.

Accepted for publication June 5, 2009

Address reprint requests to Ganesan Ramesh, Ph.D., Division of Nephrology, H040, Pennsylvania State University College of Medicine, 500 University Drive, Hershey, PA 17033. E-mail: gramesh@psu.edu. 
mice are resistant to I/R injury. This resistance was associated with reduced cytokine production, increased cell proliferation, and the suppression of apoptosis in tubular epithelial cells.

\section{Materials and Methods}

\section{Renal I/R}

C57BL/6J mice (8 to 9 weeks of age; The Jackson Lab, Bar Harbor, ME) or netrin-1 transgenic mice that express chicken netrin-1 in proximal tubular epithelial cells under control of the L-fatty acid binding protein promoter $^{16}$ were anesthetized with sodium pentobarbital $(50 \mathrm{mg} / \mathrm{kg}$ body weight, intraperitoneally (IP)) and were placed on a heating pad to maintain core temperature at $37^{\circ} \mathrm{C}$. Both renal pedicles were identified through dorsal incisions and clamped for 26 minutes. Reperfusion was confirmed visually on release of the clamps. As a control, sham operated animals were subjected to the same surgical procedure except the renal pedicles were not clamped. Surgical wounds were closed and the mice were given $1 \mathrm{ml}$ of warm saline (IP) and kept in a warm incubator until they regained consciousness.

\section{Renal Function}

Renal function was assessed by measurements of blood urea nitrogen (VITROS DT60II Chemistry Slides, OrthoClinical Diagnostics, Raritan, NJ) and serum creatinine (catalog number DZ072B, Diazyme Labs, Poway, CA).

\section{Quantitation of mRNA by Real-Time RT-PCR}

Real-time RT-PCR was performed in an Applied Biosystems, Inc. 7700 Sequence Detection System (Foster City, California). RNA (1.5 $\mu \mathrm{g}$ total) was reverse transcribed in a reaction volume of $20 \mu \mathrm{l}$ by using the Omniscript RT kit (Qiagen, Valencia, CA) and random primers. The product was diluted to a volume of $150 \mu \mathrm{l}$ and $6 \mu$ l aliquots were used as templates for amplification by using the SYBR Green PCR amplification reagent (Qiagen) and gene-specific primers. The following primer sets were used: chicken netrin-1 (forward: 5'-ATTGCCCCCTGCATAAAGAT-3'; reverse: 5'-TGGATCTGCACAGCGTAGTC-3'); mouse tumor necrosis factor $\alpha$ (forward: 5'-GCATGATCCGCGACGTGGAA-3'; reverse: 5'-AGATCCATGCCGTTGGCCAG-3'); MCP-1 (forward: 5'-ATGCAGGTCCCTGTCATG-3'; reverse: 5'-GCTTGAGGTGGTTGTGGA-3'); interferon $\gamma$ (forward: 5' TCAGCAACAGCAAGGCGAAAAAG-3'; reverse: 5'-ACCCCGAATCAGCAGCGACTC-3'); IL-6 (forward: 5'-GATGCTACCAAACTGGATATAATC-3'; reverse: 5'-GGTCCTTAGCCACTCCTTCTGTG-3'); ICAM-1 (forward: 5'-AGATCACATTCACGGTGCTG-3'; reverse: 5'-CTTCAGAGGCAGGAAACAGG-3'); VCAM-1 (forward: 5'-ATTTTCTGGGGCAGGAAGTT-3'; reverse: 5'-ACGTCAGAACAACCGAATCC-3'); E-selectin (forward: 5'-AGCTACCCATGGAACACGAC-3'; reverse: 5'-ACGCAAGTTCTCCAGCTGTT-3'); netrin-1 (forward: 5'-AAGCCTATCACCCACCG-
GAAG-3'; reverse: 5'-GCGCCACAGGAATCTTGATGC-3'); netrin-4 (forward: 5'-AACAGGGGCTCCTAACGAAT-3'; reverse: 5'-GTCTTCTGAGGTCGCGGTAG-3'); UNC5A (forward: 5'-ATCCCTAACACAGGAATCAGC-3'; reverse: 5'CTAACGATAGGACTCAGCAGG-3'); UNC-5B (forward: 5'TGGATCTTTCAGCTCAAGACCCAG-3'; reverse: 5'-AAGATGGCCAGCTGGAGCCG-3'); UNC5C (forward: 5'GATGAAACCTCTGGTCTAATTGTG-3'; reverse: 5'-CCTTCCGACTCTTCGTAGTG-3'); UNC5D (forward: 5'-GTGAACATCTTCGTATCCGT-3'; reverse: 5'-TTCTCAATGCCTCTCCTACTC-3'); DCC (forward: 5'-CTCTTCACAGGATTGGAGAAAGGC-3'; reverse: 5'-GAGGAGGTGTCCAACTCATGATG-3'); and netrin-3 (forward: 5'-TGGCTGGTTGACTTACAGCGG-3'; reverse: 5'-TACAAGAGCGAGGCTCCCTCG-3'). The amount of DNA was normalized to the B-actin signal amplified in a separate reaction (forward primer: 5'-AGAGGGAAATCGTGCGTGAC-3'; reverse: 5'-CAATAGTGATGACCTGGCCGT-3').

\section{Western Blot Analysis}

Protein was extracted by placing the kidney in a radioimmunoprecipitation assay buffer containing a protease and phosphatase inhibitor cocktail (Sigma Chemical, Co., St. Louis, MO). Protein concentration was quantitated by using the BCA protein assay reagent (Pierce Biotechnology, Inc., Rockford, IL) and $50 \mu \mathrm{g}$ of total protein was loaded onto 4 to $12 \%$ polyacrylamide gels, separated, and then transferred onto a polyvinylidene difluoride membrane. The membrane was probed with goat anti-chicken netrin-1 antibody (R\&D Systems, Minneapolis, MN), goat anti-mouse netrin-1 antibody (Santa Cruz Biotechnology, Santa Cruz, CA), and mouse anti- $\beta$ actin antibody (Sigma Chemical Co.). Antibodies against chicken and mouse netrin-1 do not cross-react with different species netrin-1. Proteins were detected by using enhanced chemiluminescence detection reagents (Amersham Pharmacia Biotech, Inc., Pittsburgh, PA). To detect urine netrin-1, $10 \mu \mathrm{g}$ creatinine equivalent of urine was loaded onto polyacrylamide gel and Western blot analysis was performed as described above.

\section{Immunohistochemical Localization of Chicken Netrin-1, Caspase-3, and Ki-67}

Immunohistochemical localization of chicken netrin-1 was performed as described before ${ }^{10}$ with modification. Briefly, the kidneys were perfused with PBS and fixed overnight in 4\% paraformaldehyde and then transferred to $30 \%$ sucrose. The kidney was placed in a cryomold and frozen. Six-micrometer-thick sections were placed on glass slides. Sections were washed with PBS, permeabilized with $0.2 \%$ Triton $X-100$ in PBS, washed, and blocked with PBS containing 5\% donkey serum and $1 \%$ bovine serum albumin. Primary antibodies included a rabbit anti-Ki-67 (Novus Biological, Littleton, CO) and a goat anti-chicken netrin-1 polyclonal antibody (R\&D Systems). Primary antibodies were detected by using secondary antibodies conjugated with fluorescein isothio- 
cyanate (Abcam, Cambridge, MA) or Alexa Fluor 560 (Invitrogen, Carlsbad, CA). To identify specific tubular segments that express chicken netrin-1, double staining was performed in which the chicken netrin-1 antibody was detected with an Alexa-633 conjugated secondary antibody followed by incubation with fluorescein isothiocyanate-conjugated lectins. Phytohemagglutinin (PHA) E was used as marker of the proximal tubule. Slides were mounted in aqueous mounting medium (Santa Cruz Biotechnology) and viewed by using a Leica Confocal microscope (Leica Microsystems, Bannockburn, IL).

To determine caspase-3 activation, paraffin embedded sections were deparaffinized and hydrated. Endogenous peroxidase activity was blocked with $90 \%$ methanol/3\% $\mathrm{H}_{2} \mathrm{O}_{2}$ followed by permeabilization with $0.2 \%$ Triton $\mathrm{X}-100$ in PBS. Then sections were washed and blocked with PBS containing 5\% donkey serum and $1 \%$ bovine serum albumin. Primary antibodies included goat anti-UNC5B, goat anti-UNC5C, and goat anti-neogenin polyclonal antibodies (R\&D Systems). Primary antibodies were detected by using a biotin conjugated secondary antibody followed by an avidin-biotin complex reagent. Color was developed by using metal enhanced diaminobenzidine substrate. Peroxidase staining was visualized and photographed by using an inverted microscope

A

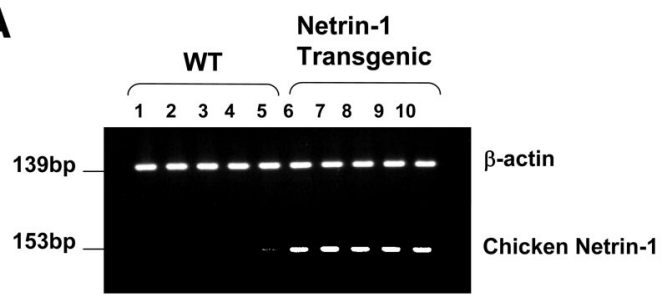

B

WT Netrin-1 transgenic

\begin{tabular}{|c|c|c|c|c|c|}
\hline \multicolumn{2}{|c|}{ IR } & \multirow{2}{*}{$\begin{array}{l}\text { Sham } \\
24 \mathrm{hr}\end{array}$} & \multicolumn{2}{|c|}{ IR } & \multirow{2}{*}{$\begin{array}{c}\text { Sham } \\
24 \mathrm{hr}\end{array}$} \\
\hline $6 \mathrm{hr}$ & $24 \mathrm{hr}$ & & $6 \mathrm{hr}$ & $24 \mathrm{hr}$ & \\
\hline
\end{tabular}

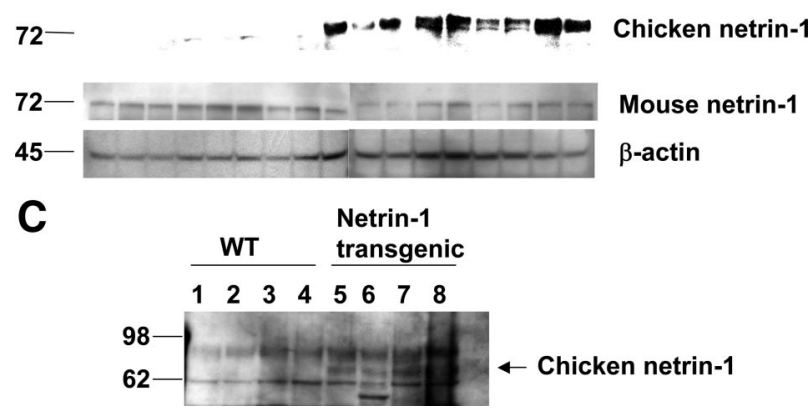

Figure 1. Netrin-1 transgene expression in the kidney. A: Real time RT-PCR analysis of chicken netrin-1 mRNA expression in the kidney from wild-type (WT) and transgenic mice. mRNA expression was seen only in transgenic animals. B: Western blot analysis of chicken netrin-1 and mouse netrin-1 protein expression in WT and transgenic animals subjected to sham surgery or I/R injury. Chicken netrin-1 protein was detectable only in the kidney from transgenic mice, whereas mouse netrin-1 is expressed in both WT and transgenic animals. The expression of chicken netrin-1 was not changed after I/R. C: Western blot analysis of chicken netrin-1 protein in urine samples from WT and netrin-1 transgenic animals. Chicken netrin-1 protein was detectable only in transgenic animal urine but not in WT animal urine.
(Olympus America, Inc., Center Valley, PA) with a CCD camera attached to it.

\section{TACS TdT in situ Apoptosis Detection}

To identify apoptotic cells, tissue sections were stained by using TACS TdT in situ Apoptosis Detection kit (R\&D Systems) according to the manufacturer's instruction. Briefly, tissue sections were deparaffinized, hydrated, and washed with PBS. Sections were digested with proteinase $\mathrm{K}$ for 15 minutes at $24^{\circ} \mathrm{C}$. Slides were then washed and endogenous peroxidase activity was quenched with $3 \% \mathrm{H}_{2} \mathrm{O}_{2}$ in methanol. Slides were washed and incubated with $\mathrm{TdT}$ labeling reaction mix at $37^{\circ} \mathrm{C}$ for 1 hour and then with streptavidin-horseradish peroxidase. Color was developed by using TACS blue label substrate solution. Slides were washed, counterstained, and mounted with Permount (Fisher Scientific, Pittsburgh, PA). Sections were photographed and labeled cells were counted and quantitated.

\section{Histology}

Kidney tissue was fixed in buffered 10\% formalin for 12 hours and then embedded in paraffin wax. For assessment of injury, 5- $\mu \mathrm{m}$ sections were stained with PAS. Acute tubular necrosis was assessed in the outer strip of the outer medulla and cortex by using a semiquantitative
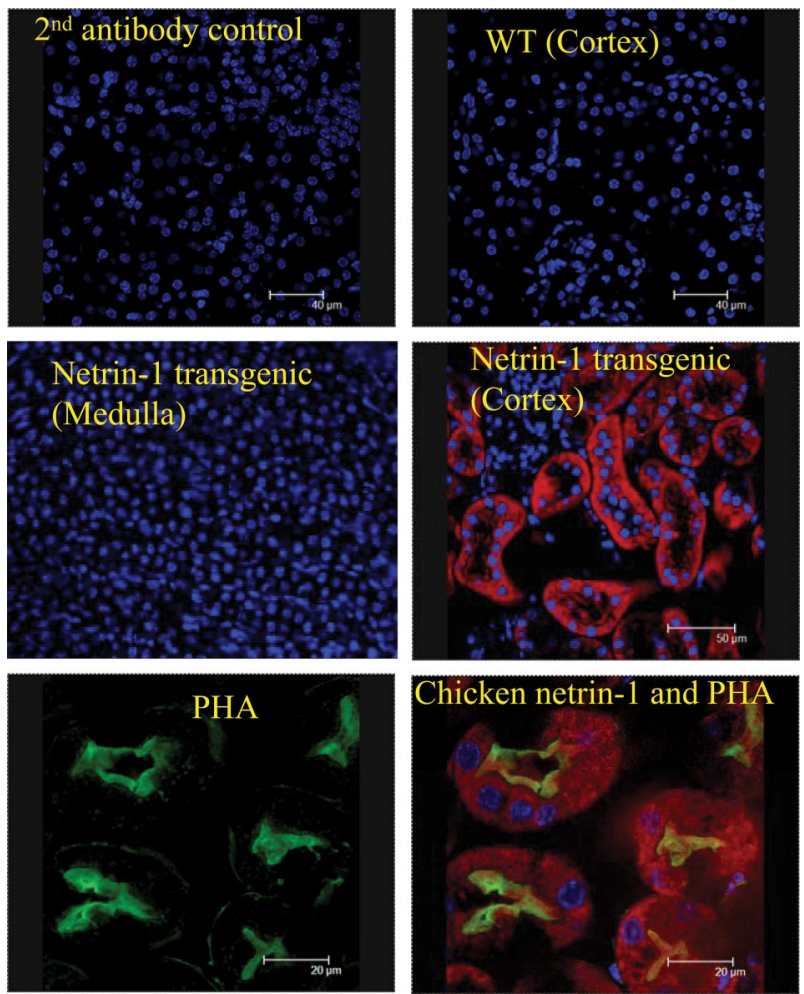

Figure 2. Localization of transgene expression. No transgene expression was seen in the kidneys from wild-type (WT) mice. Kidneys from transgenic mice showed chicken netrin-1 protein staining in the cortex. Chicken netrin-1 protein was co-localized with the proximal tubule specific lectin PHA (green). 
scale in which the percentage of tubules showing epithelial necrosis, brush-border loss, and cast formation was assigned a score: $0=$ normal; $1=<10 \% ; 2=11$ to $25 \%$; $3=26$ to $45 \%$; $4=46$ to $75 \%$; and $5=>76 \% .{ }^{17,18}$ Ten fields of $\times 40$ magnification were examined and averaged. The individual scoring the slides was blinded to the genotype of the animal. To quantitate leukocyte infiltration, sections were stained with rat anti-mouse neutrophils antibody (SEROTECH, Raleigh, NC) (1:200 dilution) followed by goat anti-rat biotin conjugate. Color was developed after incubation with an avidin-biotin complex reagent (Vector Lab, Burlingame, CA). Stained sections were photographed and five $\times 40$ fields of neutrophils were examined for quantitation of leukocytes.

\section{Statistical Methods}

All assays were performed in duplicate. The data are reported as means \pm SEM. Statistical significance was assessed by an unpaired, two tailed Student's $t$-test for single comparison or analysis of variance for multiple comparisons.

A
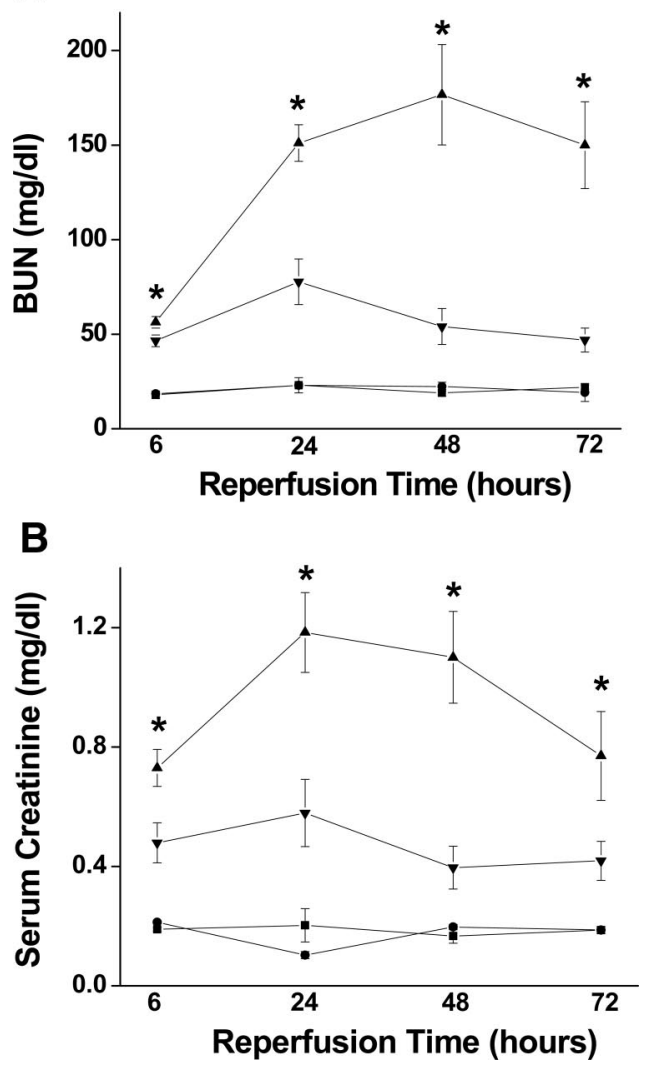

Figure 3. Effect of overexpression of netrin-1 on I/R-induced renal dysfunction. BUN (A) and serum creatinine (B) levels in sham operated wild-type (WT) (closed box), sham operated netrin-1 transgenic animals (closed circle), $\mathrm{I} / \mathrm{R}$ in WT mice (upward triangle) ${ }^{7}$ and $\mathrm{I} / \mathrm{R}$ of netrin- 1 transgenic mice (downward triangle). ${ }^{*} P<0.01$ versus netrin- 1 transgenic animals subjected to I/R. Values are mean $\pm \mathrm{SEM} ; n=8$ to 14 for each group.

\section{Results}

Overexpressed Chicken Netrin-1 is Localized in Tubular Epithelial Cells and Can Be Detected in Urine

Netrin-1 overexpressing transgenic mice were created by using the fatty acid binding protein promotor to control netrin-1 expression. The chicken netrin-1 mRNA was found to be expressed in the colon, the small intestine, and the kidney. ${ }^{16}$ However, the localization of chicken netrin- 1 in the kidney had not been determined. Therefore, we analyzed the expression of chicken netrin-1 mRNA and protein in these mice. As shown in Figure 1, chicken netrin-1 mRNA (Figure 1A) and protein (Figure 1B) are highly expressed in the kidneys of the transgenic mice but not in wild-type mice. The expression of the chicken netrin-1 is not altered after I/R. Endogenous mouse netrin-1 is expressed in both wildtype and transgenic mice at similar levels. As reported previously, ${ }^{10}$ endogenous netrin-1 protein was increased after I/R. Moreover, the chicken netrin- 1 is secreted in lumen

A
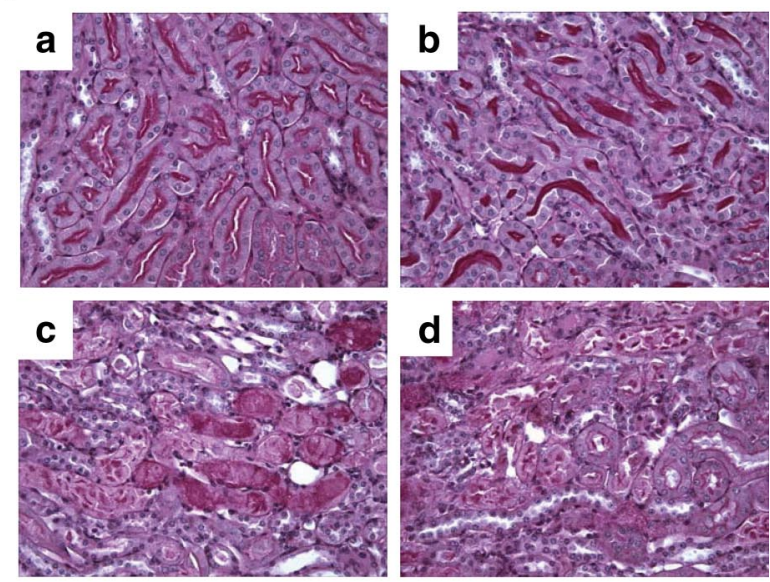

B

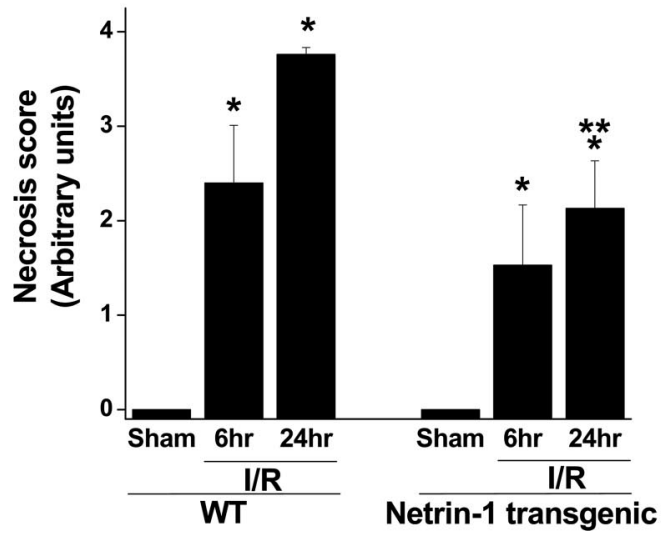

Figure 4. Renal histology in wild-type (WT) and netrin-1 transgenic mice after I/R injury. A: Tissue sections obtained 24 hours after sham surgery in WT (a) and netrin-1 transgenic mice (b) or 24 hours after I/R in WT (c) or in netrin-1 transgenic mice (d). WT mice exhibited greater renal injury in the outer stripe of outer medulla when compared with netrin-1 transgenic mice. B: Acute tubular necrosis was assessed in the juxtamedullary cortex by using a semiquantitative scale as described in Methods. ${ }^{*} P<0.01$ versus sham operated and ${ }^{* *} P<0.05$ versus WT at 24 hours of I/R. $n=4$ to 6 kidneys per genotype. 
and excreted in urine, which can be detected by Western blot (Figure 1C). Immunolocalization studies showed that the chicken netrin-1 is expressed in proximal tubular epithelial cells (Figure 2).

\section{Netrin-1 Transgenic Animals Are Resistant to I/R-Induced Renal Dysfunction}

We had previously shown that the administration of recombinant netrin-1 reduced renal ischemic injury. ${ }^{10}$ To explore the mechanism and to determine whether ne-

A
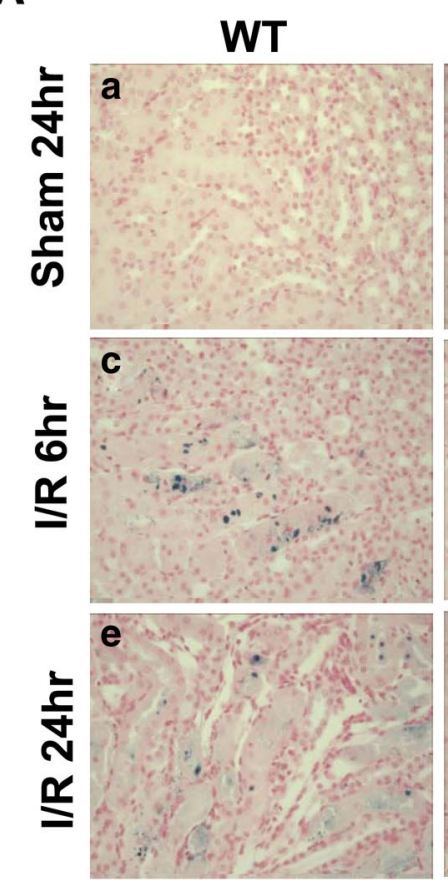

B

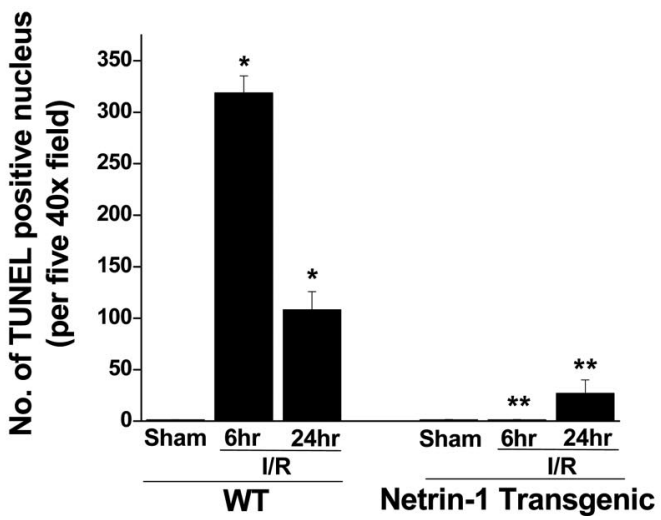

Figure 5. Effect of netrin-1 overexpression on I/R-induced apoptosis. Apoptotic cells were identified by using terminal deoxynucleotidyl transferasemediated dUTP nick-end labeling staining (a). Sham operated wild-type (WT) and transgenic animal kidney did not show any apoptotic cells (a and b). Within 6 hours after reperfusion, the number of apoptotic cells (blue staining) was increased dramatically in WT animals (c), whereas only few cells were positive for apoptosis in transgenic animals (d). Twenty-four hours after reperfusion, apoptosis was decreased but still significantly higher than sham in WT animals (e), whereas transgenic animals showed few apoptotic cells for apoptosis (f). B: Quantitation of apoptotic cells. Apoptotic positive cells were counted in 5 random $\times 40$ fields. ${ }^{*} P<0.005$ versus sham operated. ${ }^{*} *{ }^{*} P<0.001$ versus WT I/R. $N=4$ to 6 . trin-1 is acting locally to reduce injury, wild-type and netrin-1 transgenic animals were subjected to 26 minutes of bilateral renal ischemia followed by reperfusion for varying intervals. Renal function was determined by measuring blood urea nitrogen (BUN) and serum creatinine. As shown in Figure 3, A and B, wild-type mice developed severe renal dysfunction. BUN and creatinine were increased significantly by 6 hours and continued to climb up to 48 hours before starting to decrease. However, netrin-1 transgenic animals displayed lower BUN and creatinine levels. The sham operated animals did not develop any renal dysfunction in either wild-type or netrin-1 transgenic animals (Figure 3). Improved renal function in the transgenic animals was associated with improved tissue morphology as determined by reduction in necrosis score as compared with wild-type mice (Figure $4 A)$. Semiquantitative scoring of tubule injury was significantly higher in wild-type kidneys $(3.8 \pm 0.1)$ than in kidneys from netrin- 1 transgenic mice $(2.4 \pm 0.6)$ at 24 hours after I/R $(P<0.05 ; n=4$ to 6 per genotype; Figure 4B).

\section{Netrin-1 Overexpression Reduces I/R-Induced Tubular Epithelial Cell Apoptosis}

Netrin-1 was shown to be a survival factor for intestinal epithelial cells and in the absence of netrin-1, the receptors DCC and UNC5B are known to induce apoptosis. ${ }^{16,19,20} \mathrm{I} / \mathrm{R}$ is known to induce apoptosis in tubular epithelial cells. In that context, inhibition of apoptosis leads to improved renal function and prevents inflammation. ${ }^{21}$ To determine whether netrin-1 alters I/R-induced apoptosis, apoptotic cells were quantitated by using

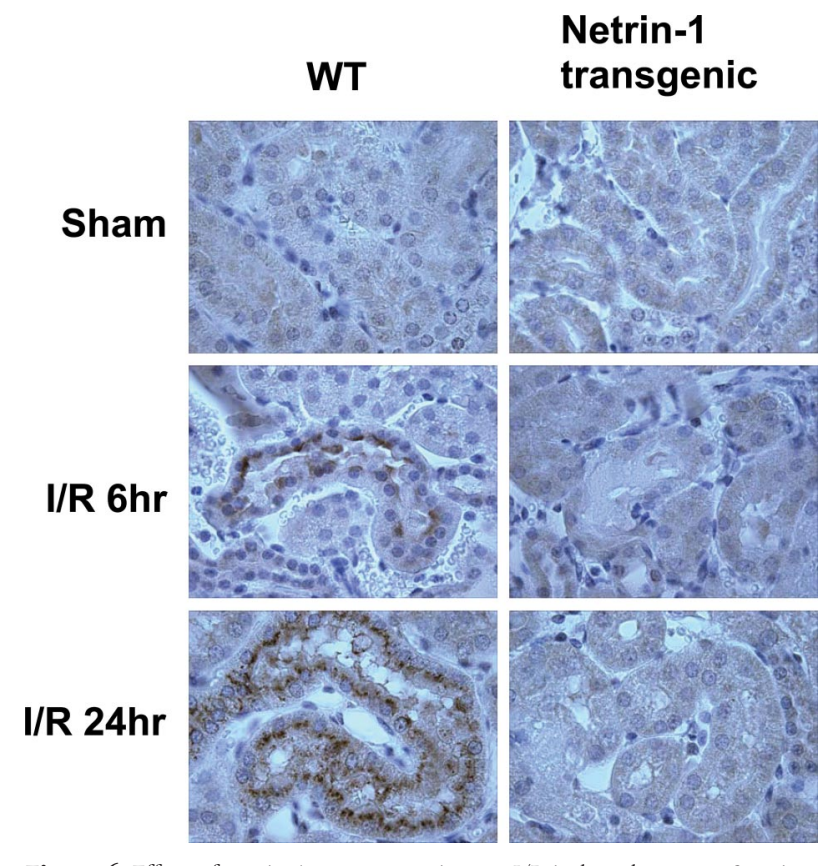

Figure 6. Effect of netrin-1 overexpression on I/R-induced caspase- 3 activation. Kidneys from Sham operated wild-type (WT) and transgenic mice did not show any staining for active caspase-3. Within 6 hours after reperfusion the number of tubular epithelial cells positive for active caspase- 3 was increased in WT mice and increased further at 24 hours after reperfusion. 
TACS TdT staining. Sham operated wild-type (Figure 5A) and netrin-1 transgenic (Figure 5B) kidneys did not show any apoptotic cells. I/R induced a large increase in apoptosis in wild-type mice by 6 hours of reperfusion (Figure $5 C$ ), which then reduced at 24 hours (Figure 5D) suggesting clearance of apoptotic cells. However, I/R-induced apoptosis was almost completely absent in netrin- 1 transgenic kidneys both at 6 hours (Figure 5E) and 24 hours (Figure 5F).

Tissue sections were also stained with antibodies against active caspase-3. As shown in Figure $6,1 / R$ increased active capase- 3 staining at 6 and 24 hours in the

\section{A}
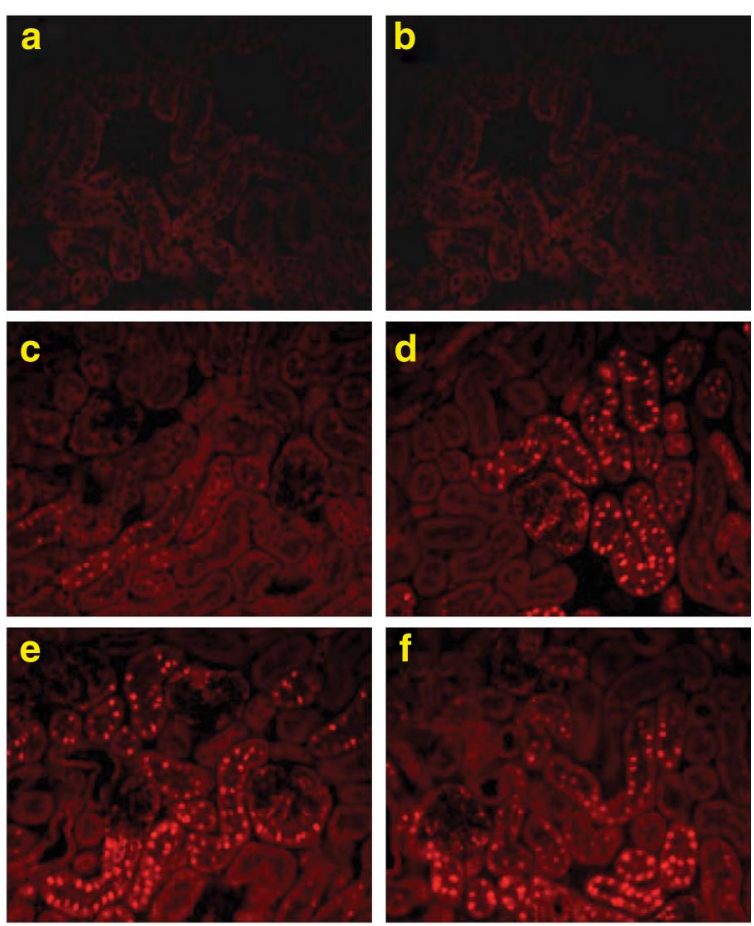

B

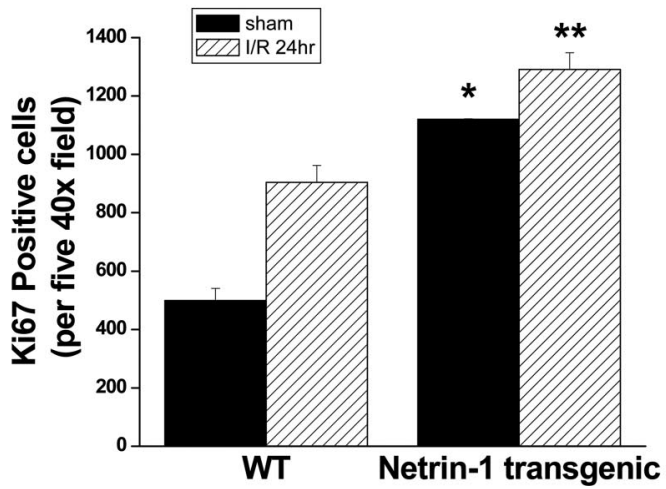

Figure 7. A: Effect of Netrin-1 overexpression on tubular epithelial cell proliferation. Proliferating tubular epithelial cells were identified by staining for Ki-67. Immunofluorescence control (secondary antibody alone) in tissue section from wild-type (WT) (a) and netrin-1 transgenic (b) mouse subjected to I/R. Sham operated WT mice kidney showed few cells positive for Ki-67 (c), whereas sham operated transgenic mice showed more cells positive for $\mathrm{Ki}-67$ (d). I/R induced a large increase in Ki-67 staining in WT mice (e) and a further increase in netrin-1 transgenic mice (f). B: Quantitative data. Ki-67 positive nuclei were counted in 5 high power fields $(\times 400)$ in each section. ${ }^{*} P<0.005$ versus WT sham operated animals. ${ }^{* *} P<0.005$ versus WT I/R 24 hours. $N=4$ wild-type mice. However, active caspase staining was absent in netrin-1 transgenic animals kidney. These results confirm the effect of netrin-1 against I/R-induced renal tubular epithelial cell apoptosis.

\section{Netrin-1 Overexpression Increases Both Basal and I/R-Induced Tubular Epithelial Cell Proliferation}

Netrin-1 is known to increase the proliferation of Schwann cells ${ }^{22}$ and endothelial cells. ${ }^{23}$ Our recent in vitro studies showed that netrin-1 increases proximal tubular epithelial cells proliferation. ${ }^{24}$ To determine whether netrin-1 increases tubular epithelial cell proliferation, kidney tissue sections were stained for the proliferation marker Ki-67. In sham operated wild-type kidney, very few cells were positive for Ki-67 (Figure 7, A-C). Staining was significantly increased after I/R (Figure 7E). In contrast, netrin-1 overexpression caused an increase in $\mathrm{Ki}-67$ positive cells in sham operated animals (Figure 7D), which was further increased at 24 hours after reperfusion (Figure 7F). The summary data in Figure 7B indicate that Netrin-1 significantly $(P<0.005$ versus wild-type) increased both basal and I/R-induced tubular epithelial cell proliferation.

\section{Netrin-1 Overexpression Reduced Cytokine Expression, Oxidative Stress, and Increased NOS Expression}

The inhibition of apoptosis is known to reduce I/R-induced inflammation. ${ }^{21}$ Moreover, netrin-1 administration reduced inflammation and inhibited leukocyte migration. ${ }^{9,10}$ To determine whether netrin-1 overexpression reduces inflammation, expression of several cytokines, chemokines, and adhesion molecules were determined by real-time RT-PCR. I/R induced a significant increase in

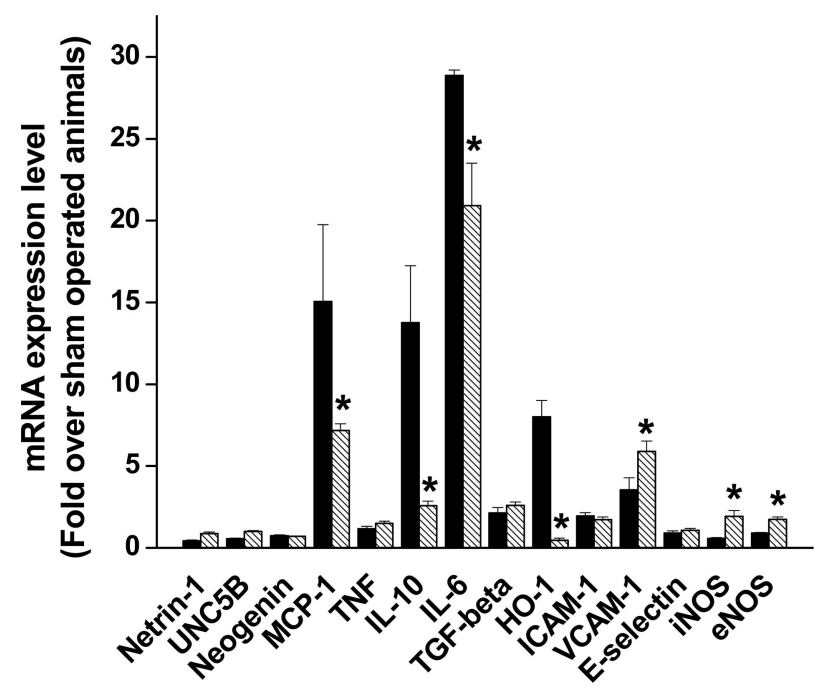

Figure 8. Effect of netrin-1 overexpression on cytokine and adhesion molecule gene expression. Effect of netrin-1 on gene expression was measured at 24 hours after sham operation and I/R of wild-type (WT) animals (solid bar) and netrin- 1 transgenic mice (hatched bar). Values are means \pm SEM ${ }^{*} P<0.05$ versus WT animals. $N=4$. 
MCP-1, IL-6, TGF- $\beta$, IL-10, ICAM- 1 , and VCAM- 1 mRNA at 24 hours (Figure 8). Overexpression of netrin-1 significantly reduced MCP-1, IL-6, IL-10, but not TGF- $\beta$, ICAM-1, and VCAM-1. In addition, ischemia induced a large increase in the oxidative stress marker hemoxygenase- 1 , which was significantly lower in netrin-1 transgenic animals subjected to I/R. Netrin-1 is known to induce eNOS expression and activity in endothelial cells. ${ }^{1}$ eNOS and iNOS expression were significantly higher in transgenic animals as compared with wild-type animals 24 hours after I/R.

\section{Netrin-1 Overexpression in the Tubular Epithelial Cells Does Not Reduce Neutrophil Infiltration}

Earlier studies suggested that intravenous administration of recombinant netrin-1 reduced neutrophil infiltration into the kidney after $\mathrm{I} / \mathrm{R}^{10}$ and leukocyte migration during sepsis. ${ }^{9}$ To determine, whether the observed protective effect of tubule specific overexpression of

\section{A}
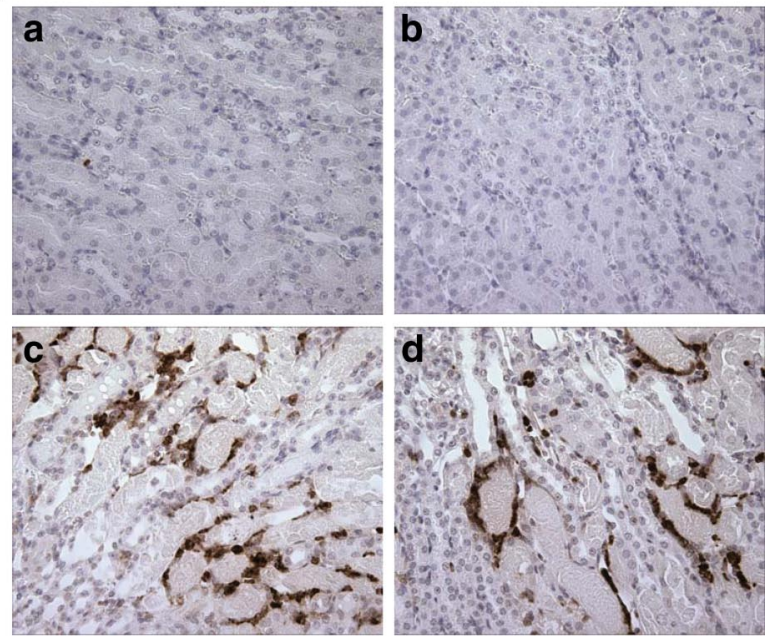

$\mathbf{B}$

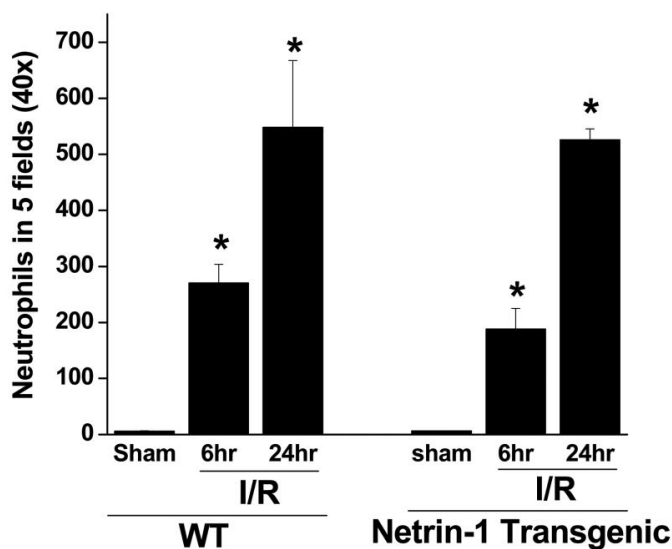

Figure 9. Effect of netrin-1 overexpression on neutrophil infiltration. Sections were fixed and stained for neutrophils as described in Materials and Methods. A: Sham-operated wild-type (WT) mice (a) and netrin-1 transgenic animals (b) showed few neutrophils. Twenty-four hours after I/R, both WT (c) and netrin-1 transgenic animals (d) had showed increased neutrophil staining. B: Quantitative summary data of neutrophil infiltration ( ${ }^{*} P<0.001$ versus sham-operated animals). $N=3$ to 6 . netrin-1 is mediated, in part, through reducing leukocyte infiltration, tissue sections were stained with an anti-neutrophil antibody (Figure 9A). Sham operated wild-type and transgenic mouse kidneys had very few neutrophils (Figure 9, A and B). I/R dramatically increased neutrophil infiltration in wild-type (Figure 9C) and in netrin-1 transgenic mice (Figure 9D). These results suggest that the observed protective effect of netrin-1 overexpression is not due to suppression of neutrophil infiltration.

\section{Discussion}

The present study provides evidence that netrin-1, a laminin related neuronal guidance cue, when expressed in proximal tubule cells can prevent renal dysfunction and epithelial cell apoptosis in response to ischemia. Furthermore, netrin- 1 increased the proliferation of tubular epithelial cells and reduced inflammation. These results indicate that netrin- 1 not only plays a key role in neuronal development but also has a key role outside the nervous system, including in the kidney.

The transgene, chicken netrin-1, was highly expressed in proximal tubular epithelial cells. The presence of chicken netrin-1 in the urine suggests that it is secreted into the lumen. Whether netrin-1 is secreted only into the lumen or also secreted basally is not clear. The kidney function and histology of the transgenic mice appears normal, suggesting that netrin-1 expression does not influence normal kidney function.

Our earlier studies had showed that netrin-1 expression is highly induced in renal tubular epithelial cells and down-regulated in endothelial cells in vivo after I/R injury of the kidney. ${ }^{10}$ In addition, the administration of exogenous netrin-1 results in reduced inflammation and improved renal function. However, the role of netrin-1 expressed in tubular epithelial cells and its contribution to renal function was not known. The present study determined that netrin-1 overexpression protected against I/Rinduced renal failure. This effect is likely due to the marked suppression of apoptosis in the transgenic mice. Although, several in vitro and in vivo studies in other tissues have demonstrated a role for netrin- 1 in inhibiting apoptosis, ${ }^{8,12,15,16,25}$ this is the first study to show the anti-apoptotic role of netrin-1 in kidney tubules.

Netrin-1 receptors (DCC and UNC5) are also called dependence receptors as these receptors are known to induce apoptosis when they are not bound to netrin- $1 .{ }^{19}$ The pathways through which unoccupied netrin- 1 receptors mediate apoptosis are not well understood. Caspase mediated cleavage of the intracellular domain of UNC5H or DCC receptors is reported to lead to the release of cytoplasmic addiction dependence domains and activation of apoptotic pathways. ${ }^{11,12}$ These pathways include activation of caspase- $9,{ }^{14}$ activation of DAPK, ${ }^{26}$ or NRAGE. ${ }^{27}$ Cell death induced by unbound UNC5H2 has been shown to be dependent on p53. ${ }^{28}$ Along this line, netrin-1 administration prevented p53 activation and ischemia-induced apoptosis in the brain. ${ }^{15}$ Both caspases and p53 are activated during I/R of the kidney 
and are known to mediate kidney injury. ${ }^{29-32}$ Since, netrin-1 is known to suppress p53 and caspase activation in other tissues, ${ }^{14,15,28}$ this suggests that a similar mechanism may exist in the kidney as well. Our results support the finding that netrin-1 suppressed caspase-3 activation (Figure 6).

Netrin-1 regulates angiogenesis, endothelial cell proliferation and migration, the development of mammary glands, and also intestinal crypt epithelial cell survival and apoptosis. ${ }^{1,3,16}$ Earlier studies using transgenic mice that overexpress netrin-1 in intestinal crypt epithelial cells showed that netrin-1 expression did not induce proliferation but inhibited apoptosis. ${ }^{16}$ In our study, a large increase in epithelial cell proliferation was observed under basal conditions. The mechanism underlying this difference is not clear. However, we previously reported that netrin-1 stimulates tubular epithelial cell proliferation in vitro in an ERK and Akt-dependent fashion. ${ }^{24}$ Netrin-1 is also known to induce eNOS expression and production of nitric oxide through ERK pathways. ${ }^{1}$ We found that netrin-1 transgenic mice had increased expression of eNOS and iNOS mRNA as compared with wild-type control. However, the contribution of eNOS and iNOS to netrin-1 mediated prevention of renal injury needs further study.

Inflammatory mechanisms play a key role in I/R injury of the kidney. ${ }^{33-35}$ Recent studies suggest that netrin-1 limits inflammation by inhibiting leukocyte migration via UNC5B receptors. ${ }^{9}$ Although intravenous administration of netrin-1 reduced I/R-induced neutrophil infiltration, tubule specific overexpression in the present study did not influence neutrophil infiltration. This observation suggests that the observed protective effects in netrin-1 transgenic mice against $I / R$ injury is not through suppression of neutrophil infiltration. It is possible that the netrin-1 expressed in proximal tubule cells may not be available to the vascular compartment to act on the leukocytes. However, netrin-1 transgenic mice did show a reduction in certain pro-inflammatory chemokines and cytokines, notably MCP-1 and IL-6. Since inhibition of MCP-1 and IL-6 have both been shown to reduce renal ischemic injury, some of the protection afforded by netrin-1 could be referable to this reduction.

In summary, our findings support the view that netrin-1 is a strong prosurvival factor for proximal tubule cells, can suppress I/R-induced apoptosis, possibly by suppressing caspase-3 activation, and increases epithelial cell proliferation. In addition, netrin-1 reduces oxidative stress and cytokine expression and increases eNOS and iNOS expression, suggesting that netrin-1 may be a useful therapeutic molecule for treating ischemic kidney disease. The molecular mechanism by which netrin-1 suppresses apoptosis warrants further investigation.

\section{References}

1. Nguyen A, Cai H: Netrin-1 induces angiogenesis via a DCC-dependent ERK1/2-eNOS feed-forward mechanism. Proc Natl Acad Sci USA 2006, 103:6530-6535

2. Wilson BD, li M, Park KW, Suli A, Sorensen LK, Larrieu-Lahargue F Urness LD, Suh W, Asai J, Kock GA, Thorne T, Silver M, Thomas KR,
Chien CB, Losordo DW, Li DY: Netrins promote developmental and therapeutic angiogenesis. Science 2006, 313:640-644

3. Navankasattusas S, Whitehead KJ, Suli A, Sorensen LK, Lim AH Zhao J, Park KW, Wythe JD, Thomas KR, Chien CB, Li DY: The netrin receptor UNC5B promotes angiogenesis in specific vascular beds. Development 2008, 135:659-667

4. Hebrok M, Reichardt LF: Brain meets pancreas: netrin, an axon guidance molecule, controls epithelial cell migration. Trends Cell Biol 2004, 14:153-155

5. Liu Y, Stein E, Oliver T, Li Y, Brunken WJ, Koch M, Tessier-Lavigne M, Hogan BLM: Novel role for netrins in regulating epithelial behavior during lung branching morphogenesis. Curr Biol 2004, 14:897-905

6. Lu X, Le Noble F, Yuan L, Jiang Q, De Lafarge B, Sugiyama D, Breant C, Claes F, De Smet F, Thomas JL, Autiero M, Carmeliet P, TessierLavigne M, Eichmann A: The netrin receptor UNC5B mediates guidance events controlling morphogenesis of the vascular system. Nature 2004, 432:179-186

7. Arakawa $\mathrm{H}$ : Netrin-1 and its receptors in tumorigenesis. Nat Rev Cancer 2004, 4:978-987

8. Fitamant J, Guenebeaud C, Coissieux MM, Guix C, Treilleux I, Scoazec JY, Bachelot T, Bernet A, Mehlen P: Netrin-1 expression confers a selective advantage for tumor cell survival in metastatic breast cancer. Proc Natl Acad Sci USA 2008, 105:4850-4855

9. Ly NP, Komatsuzaki K, Fraser IP, Tseng AA, Prodhan P, Moore KJ Kinane TB: Netrin-1 inhibits leukocyte migration in vitro and in vivo. Proc Natl Acad Sci USA 2005, 102:14729-14734

10. Wang W, Brian RW, Ramesh G: Netrin-1 and kidney injury. I. Netrin-1 protects against ischemia-reperfusion injury of the kidney. Am $J$ Physiol Renal Physiol 2008, 294:F739-F747

11. Mehlen P, Rabizadeh S, Snipas SJ, Assa-Munt N, Salvesen GS Bredesen DE: The DCC gene product induces apoptosis by a mechanism requiring receptor proteolysis. Nature 1998, 395:801-804

12. Llambi F, Causeret F, Bloch-Gallego E, Mehlen P: Netrin-1 acts as a survival factor via its receptors UNC5H and DCC. EMBO J 2001, 20:2715-2722

13. Furne C, Rama N, Corset V, Chedotal A, Mehlen P: Netrin-1 is a survival factor during commissural neuron navigation. Proc Natl Acad Sci USA 2008, 105:14465-14470

14. Forcet C, Ye X, Granger L, Corset V, Shin H, Bredesen DE, Mehlen P: The dependence receptor DCC (deleted in colorectal cancer) defines an alternative mechanism for caspase activation. Proc Natl Acad Sci USA 2001, 98:3416-3421

15. Wu TW, Li WW, Li H: Netrin-1 attenuates ischemic stroke-induced apoptosis. Neuroscience 2008, 156:475-482

16. Mazelin L, Bernet A, Bonod-Bidaud C, Pays L, Arnaud S, Gespach C, Bredesen DE, Scoazec JY, Mehlen P: Netrin-1 controls colorectal tumorigenesis by regulating apoptosis. Nature 2004, 431:80-84

17. Wang W, Li C, Summer SN, Falk S, Wang W, Ljubanovic D, Schrier RW: Role of AQP1 in endotoxemia-induced acute kidney injury. Am J Physiol Renal Physiol 2008, 294:F1473-F1480

18. Ramesh G, Zhang B, Uematsu S, Akira S, Reeves WB: Endotoxin and cisplatin synergistically induce renal dysfunction and cytokine production in mice. Am J Physiol Renal Physiol 2007, 293:F325-F332

19. Bredesen DE, Mehlen P, Rabizadeh S: Apoptosis and dependence receptors: a molecular basis for cellular addiction. Physiol Rev 2004 , 84:411-430

20. Mehlen P, Bredesen DE: The dependence receptor hypothesis. Apoptosis 2004, 9:37-49

21. Daemen MA, van V, Denecker G, Heemskerk VH, Wolfs TG, Clauss $\mathrm{M}$, Vandenabeele $\mathrm{P}$, Buurman WA: Inhibition of apoptosis induced by ischemia-reperfusion prevents inflammation. J Clin Invest 1999, 104:541-549

22. Lee HK, Seo IA, Seo E, Seo SY, Lee HJ, Park HT: Netrin-1 induces proliferation of Schwann cells through Unc5b receptor. Biochem Biophys Res Commun 2007, 362:1057-1062

23. Yang $Y$, Zou L, Wang Y, Xu KS, Zhang JX, Zhang JH: Axon guidance cue Netrin-1 has dual function in angiogenesis. Cancer Biol Ther 2007, 6:743-748

24. Wang W, Reeves WB, Ramesh G: Netrin-1 increases proliferation and migration of renal proximal tubular epithelial cells via the UNC5B receptor. Am J Physiol Renal Physiol 2009, 296:F723-F729

25. Fan Y, Shen F, Chen Y, Hao Q, Liu W, Su H, Young WL, Yang GY: Overexpression of netrin-1 induces neovascularization in the adult mouse brain. J Cereb Blood Flow Metab 2008, 28:1543-1551 
26. Llambi F, Calheiros-Lourenco F, Gozuacik D, Guix C, Pays L, Del Rio G, Kimchi A, Mehlen P: The dependence receptor UNC5H2 mediates apoptosis through DAP-kinase. EMBO J 2005, 24:1192-1201

27. Williams ME, Strickland P, Watanabe K, Hinck L: UNC5H1 induces apoptosis via its juxtamembrane region through an interaction with NRAGE. J Biol Chem 2003, 278:17483-17490

28. Tanikawa C, Matsuda K, Fukuda S, Nakamura Y, Arakawa H: p53RDL1 regulates p53-dependent apoptosis. Nat Cell Biol 2003, 5:216-223

29. Zhang X, Zheng X, Sun H, Feng B, Chen G, Vladau C, Li M, Chen D, Suzuki M, Min L, Liu W, Garcia B, Zhong R, Min WP: Prevention of renal ischemic injury by silencing the expression of renal caspase 3 and caspase 8. Transplantation 2006, 82:1728-1732

30. Megyesi J, Udvarhelyi N, Safirstein RL, Price PM: The p53-independent activation of transcription of p21 WAF1/CIP1/SDI1 after acute renal failure. Am J Physiol Renal Physiol 1996, 271: F1211-F1216

31. Dagher PC: Apoptosis in ischemic renal injury: roles of GTP depletion and p53. Kidney Int 2004, 66:506-509

32. Devarajan P: Update on mechanisms of ischemic acute kidney injury J Am Soc Nephrol 2006, 17:1503-1520

33. Bonventre JV, Zuk A: Ischemic acute renal failure: an inflammatory disease? Kidney Int 2004, 66:480-485

34. Burne-Taney MJ, Kofler J, Yokota N, Weisfeldt M, Traystman RJ, $\mathrm{Rabb} \mathrm{H}$ : Acute renal failure after whole body ischemia is characterized by inflammation and T cell-mediated injury. Am J Physiol Renal Physiol 2003, 285:F87-F94

35. Wu H, Chen G, Wyburn KR, Yin J, Bertolino P, Eris JM, Alexander SI, Sharland AF, Chadban SJ: TLR4 activation mediates kidney ischemia/reperfusion injury. J Clin Invest 2007, 117:2847-2859 